\title{
Game Theoretic Derivations of Competitive Strategies in Conjoint Analysis
}

\author{
S. CHAN CHOI* \\ Graduate School of Management \\ Rutgers University \\ 180 University Ave. \\ New'ark, NJ 07102-1895 \\ WAYNE S. DESARBO \\ Marketing and Statistics Depts. \\ School of Business Administration \\ University of Michigan \\ Ann Arbor, MI 48109
}

[May 1993]

Key words: Product Design, Product Competition, Conjoint Analysis, Game Theory

\begin{abstract}
While conjoint analysis has been applied in a wide variety of different contexts in Marketing, most applications fail to explicitly consider retaliatory reactions from competitors. In this paper, a methodological extension is developed for conjoint analysis by explicitly modeling competition in a game theoretic context. The Nash equilibrium concept is employed to model competitive reactions to produce design, and its implications for reactive product strategies are discussed. The optimal product design problem for each firm is formulated as a nonlinear integer programming problem, which is solved via a specialized branch and bound method combined with a heuristic. In order to compute a Nash equilibrium, a sequential iterative procedure is proposed. The proposed procedure is illustrated under several scenarios of competition using previously published conjoint data.
\end{abstract}

The modeling of consumer preferences among multiattribute alternatives has been a primary concern in marketing research. In the past two decades, no technique has received more attention toward this end than conjoint analysis (Green and Rao 1971). Legions of journal pages have been devoted to both theoretical advances (for a review, see Green and Srinivasan 1978, 1990) and practical applications that have provided marketing managers with guidelines for product design (Green and Krieger 1992), market segmentation (Green and DeSarbo 1979), and product line optimization (Kohli and Sukumar 1990). Wittink and Cattin (1989)

*This research has been supported by the Henry Rutgers Research Fellowship, Rutgers University. 
have provided a perspective of the wide use of conjoint analysis based on a survey of more than 1,000 commercial applications.

Yet, few conjoint models and applications have explicitly accounted for competitive reactions by incumbent firms to a new product introduction and the resulting impact on profits. This paucity is a sharp contrast with several models of competition that have been recently developed using continuous multidimensional product spaces. For example, Carpenter (1989), Choi, DeSarbo, and Harker (1990, 1992), and DeSarbo and Rao (1986) have proposed competitive equilibrium models for multidimensional representations arising from internal or external multidimensional scaling spaces. In conjoint analysis, on the other hand, attributes are typically discrete (or categorized), and the resulting utility functions consequentially lack continuity and differentiability. This irregularity causes an inherent difficulty in applying equilibrium analysis to conjoint-based productprice design models. Instead, initial conjoint-based models have used ad hoc procedures within buyer choice simulators to account for effects of competitive reactions. In order to properly simulate competitive actions and reactions, however, such simulators would have to be run in a repetitive, cyclical fashion for each competitor to attain his "best" competitive response against the newest entries. This cycle would have to be repeated several times, and the convergence properties of such a process are unknown.

Another set of related conjoint-based product designing methods involve mathematical programming techniques for optimal product (line) positioning (e.g., Zufryden 1982; Green and Krieger 1985; Green, Carroll, and Goldberg 1981; Kohli and Sukumar 1990; Dobson and Kalish 1993). Here, some "best" subset of new product offerings for a given manufacturer are found through combinatorial optimization procedures. Again, due to the discrete nature of the conjoint attributes, equilibrium analysis has not been attempted thus far.

In this paper, we propose an alternative mathematical programming approach of product optimization, which incorporates competitors' reactions in a game theoretic context by extending the numerical equilibrium analysis of Choi, DeSarbo, and Harker $(1990,1992)$. We attempt to find a set of specific conjoint design attribute levels that are optimal with respect to some designated objective function in a Nash equilibrium framework. In this model, a firm's objective can be either profit or market share maximization, or any combination of the two.

The next section presents an equilibrium model based on a conjoint utility function, and discusses the existence of an equilibrium solution and its characteristics. Also, two iterative procedures to find Nash equilibria are discussed. Section 2 presents an algorithm to solve the nonlinear integer programming problem using a branch and bound method that exploits the special problem structure. In section 3 , we illustrate the flexibility of the methodology with conjoint data collected by Green (1978) for a major U.S. automobile tire manufacturer, where several competitive scenarios are analyzed. Finally, the last section of the paper presents a discussion of future applications and delineates further research areas, 


\section{The equilibrium model}

\subsection{The profit function}

Once the individual conjoint utility functions are estimated, most conjoint-based product positioning methodologies employ choice simulators which evaluate a prespecified set of product designs. A typical conjoint computer software simulates market shares using several choice rules such as logit, BTL, and the Maximum Utility models. In the choice model literature, a deterministic choice model is recommended for high involvement decisions such as durable goods, and a probabilistic model for low involvement decisions such as frequently purchased consumer goods (Shocker and Srinivasan 1979).

While such simulation procedures can be appealing because of their relative simplicity, they rely heavily on a scenario that prespecifies a relatively small set of "desirable" brand attributes. When the number of alternative product profiles is large, an optimization procedure becomes more efficient than simulations (i.e., exhaustive search) in finding an "optimal" profile. Although the optimization approaches tend to lead to more difficult numerical problems to solve (i.e., combinatorial problems), recent developments in heuristics approach made the optimization procedure more attractive and practical (e.g., Green and Krieger 1985; Kohli and Sukumar 1990; Dobson and Kalish 1993).

In this paper, we assume profit maximizing firms, although the proposed methodology is sufficiently flexible to accommodate any user specified objective function (including market share). The computational effort of a product optimization model using conjoint analysis depends on the individual choice model employed. Under a deterministic choice rule, the computational burden becomes excessive because of the huge number of integer variables generated, and it is impractical to solve these combinatorial problems without heuristics. When a probabilistic choice rule is employed, the number of integer variables can be reduced, but the nonlinearity of the objective function increases, and a global optimum cannot be guaranteed. However, a local optimal solution can be computed efficiently with any general nonlinear programming code.

This paper employs the logit choice model, and assumes single-brand firms. Generalization to multiple-brand firms is straightforward, but it increases the size of each optimization problem accordingly. Then, the product optimization problem for firm/ brand $j$ becomes the following nonlinear-integer programming problem:

$$
\operatorname{Max}_{v_{j}} \mathrm{II}_{j}=\left(p_{j}-c\left(x_{j}\right)\right) \sum_{i=1}^{l} \frac{\exp \left(\beta U_{i j}\right)}{\sum_{n=1}^{J} \exp \left(\beta U_{i n}\right)},
$$

subject to

$$
\begin{array}{ll}
\sum_{l=1}^{l_{k}} x_{j k l}=1, & k=1, \cdots, K, \\
x_{j k l}=0 \text { or } 1, & k=1, \cdots, K \text { and } l=1, \cdots, L_{k},
\end{array}
$$


where

$i=1, \cdots, I$ consumers;

$j=1, \cdots, J$ brands (or equivalently, firms);

$p_{j}=$ price of brand $j$

$x_{j}=$ a $(0,1)$ design vector for brand $j$ in a feasible vector space $\mathbf{X}_{j}$;

$x_{j k l}=$ is an element of $\mathbf{x}_{j}$ such that it equals 1 if brand $j$ has attribute $k$ at level $l, 0$ otherwise;

$c\left(\mathbf{x}_{j}\right)=$ cost of producing brand profile $\mathbf{x}_{j} ;$

$\beta=$ a logit parameter inversely related to the error term (i.e., $\varepsilon_{i j}$ below).

$U_{i, i}$ is consumer $i$ 's predicted conjoint utility for brand $j$ :

$$
U_{i j}=\sum_{k=1}^{K} \sum_{i=1}^{L_{k}} \lambda_{i k l} x_{j k i}+\varepsilon_{i j}
$$

where

$k=1, \cdots, K$ attributes;

$l=1, \cdots, L_{k}$ levels of attribute $k$;

$\lambda_{i k l}=$ consumer $i$ 's part-worth for level $l$ of attribute $k$; and

$\varepsilon_{i j}=$ an error term.

This conjoint utility function is assumed to be a part-worth model with main effects only, although interaction terms may be introduced without loss of generality. Also, a mixed conjoint utility model that combines part-worth models for discrete variables and ideal-point or vector models for continuous variables can be employed in our nonlinear programming formulation. Such a problem can be solved more easily since fewer integer variables are needed. Note that as $\beta$ increases, the model behaves like the Maximum Utility model, while as $\beta$ approaches zero, it becomes a uniform choice model. When appropriate, we can also introduce a "no choice" term in the denominator with a status quo utility (see Choi, DeSarbo, and Harker 1990).

Several issues need to be discussed at this point. First, our methodology can allow for individual specific $\beta$, parameters given differential error variability common in such data. Second, if market segments were known in advance, we could perform such analysis by market segment (e.g., DeSarbo, Wedel, Vriens, and Ramaswamy 1992), although substantially more complex formulations would be required in the case of a brand offered to multiple market segments. Third, in order to keep the formulation simple, we initially assume "costless repositioning" above and beyond differentiated attribute costs. Our framework can easily accommodate this problem by, for example (with continuous attributes), specifying cost as a function of "distance" from the original position (Choi, DeSarbo, and Harker 1992). Fourth, we assume that individual part-worths have already been measured 
via any conjoint procedure, including hybrid conjoint analysis or adaptive conjoint analysis. Finally, obtaining accurate attribute cost estimation remains a fundamental issue to be resolved in most conjoint analysis-based product design methodologies. In this paper, we assume that direct costs of manufacturing and distribution can be estimated at the individual attribute level as in Green and Krieger (1992) and Dobston and Kalish (1993).

\subsection{The Nash equilibrium}

Given the profit function (1) for each firm, a Nash equilibrium is a brand strategy vector $\mathbf{x}=\left[\mathbf{x}_{i}\right]_{j=1}, \ldots, J$ at which no firm can make a higher profit by changing its product strategy unilaterally. Here, product strategy is enacted through direct manipulation of the levels of the conjoint design attributes. Therefore, a Nash equilibrium is a simultaneous solution of model (1) for all the competing firms.

While the existence and uniqueness issues of equilibria have been studied extensively for a game in a continuous strategy space (see Friedman 1989), they have not been generalized for a game in a discrete strategy space such as the one analyzed here. Therefore, they need to be investigated empirically, unless the concept of a mixed strategy is employed. In reality, however, a firm would not throw a die to decide on a new product feature as implied by the mixed strategy concept. This mixed strategy concept has often "come under heavy fire" (Rubinstein 1991), and will not be considered here.

Among many methods to compute Nash equilibria are two iterative procedures using tatonnement sequences: simultaneous and sequential tatonnements. The former.assumes that all players select respective strategies simultaneously, while the latter specifies a predetermined order of movement that allows players to select strategies in turn. We believe that the sequential moves are more representative of the real market, since it is rare to observe all firms move simultaneously, each without knowing the others' move. In addition, the latter procedure has a better chance of finding an equilibrium if one exists.

We note several characteristics of a discrete Nash equilibrium and the iterative procedure. First, it is difficult to verify the existence of an equilibrium, and we cannot guarantee that either iteration procedure finds an equilibrium even if one exists. Depending on the payoff matrix configuration, a tatonnement process may cycle without ever finding an equilibrium. Second, the iterative procedure finds at most one equilibrium even when there are many. The potential number of multiple equilibria may increase as the problem size becomes larger, since possible strategy combinations increase exponentially. Also, the equilibrium solution found may be very sensitive to the starting position. Finally, in the sequential tatonnement, an equilibrium solution is sensitive to the order of movement. Even so, it can be shown that the first firm to move is not always advantageous. In summary, when there are many equilibria, the solution to be found by an iterative procedure depends on the particular scenario of the initial position and the order 
of movement. Therefore, it is beneficial to perform a variety of separate analyses involving different assumptions concerning initial position, order of movement, etc. to examine the robustness of the resulting solutions.

\section{A computational procedure for finding a Nash equilibrium solution in conjoint analysis}

This section describes a computational procedure for the equilibrium problem defined in the previous section. First, we present a branch and bound procedure coupled with a heuristic to solve the Seller's profit maximization problem of model (1), which is formulated as a nonlinear integer programming problem. We then briefly discuss the properties of the proposed iterative procedure in finding a Nash equilibrium.

\subsection{A branch and bound method}

In solving problem $(1)$, an implicit enumeration procedure for $(0,1)$ variables would require $R=\sum_{k=1}^{K} L_{k}$ branch levels (depth) with two branches emanating from each node. However, our procedure exploits the structure of the constraints (2) and (3) to reduce the depth of branches to $K$ (number of attributes) with $L_{k}$ (number of levels) branches per node. That is, we explicitly imbed the constraints (2) in the branching algorithm, by examining $L_{k}$ branches emanating from a single node, reducing the depth to $K$.

The branching strategy can be made more efficient by using a particular heuristic. That is, finding a "good" integer solution quickly can help fathoming other nodes without further branching. Then, the questions become (a) which attribute should be branched first, and (b) which level should be examined first to provide a "good" initial lower bound. Since the objective function in problem (1) is highly nonlinear, it is difficult to directly apply the "seller's greedy heuristic" (Green and Krieger 1985). Therefore, we propose a heuristic similar to the "buyer's greedy" principle in maximizing the number of buyers rather than profit. This procedure is more appropriate when the differences in unit returns among attributes are relatively small (see Green and Krieger 1992). For our problem, this would imply branching into an attribute that has higher importance ratings across respondents (i.e., self-explicated ratings in hybrid conjoint analysis or ranges in part-worths in conventional full profile analysis). The same strategy may be applied to selecting the level to be evaluated first within an attribute. However, the level selection problems are somewhat more difficult since more preferred attribute levels tend to cost more.

As usual, a node is fathomed whenever its value (which is an upper bound for its descendent nodes) is below the lower bound. The best integer solution found in the previous nodes provides a lower bound, which is updated whenever a better 
integer solution is found. This branch and bound process continues until all nodes are fathomed. Because of the nonlinearity of the objective function, however, the bounds in the branch and bound process can be applied only locally. That is, these bounds are not to be interpreted as the global bounds of the problems. Therefore, as in any combinatorial procedure, this procedure intends to find a "good" locally optimal solution.

\subsection{Finding a Nash equilibrium}

Based on the proposed algorithms described above, an equilibrium solution can be found using an iterative process that successively solves the Seller's profit maximization problem. As discussed in the previous section, this paper focuses on the sequential tatonnement process to find an equilibrium. We assume that the order of play is given exogenously. That is, when a firm is about to introduce a new product, the order of move for the rest is assumed already set. While it may be a strong assumption, a manager should be able to approximate the order through managerial judgment, past experience, and other sources of information.

In a sequential game, firms play in turn, observing others' moves. That is, a firm selects its optimal product profile with perfect information (Friedman 1990, p. 29). This process continues until no firm wants to alter its product strategy, which is a (local) Nash equilibrium. As in the general case, existence and uniqueness of an equilibrium are difficult to establish. It may not be possible to prove theoretically that this process always leads to an equilibrium solution even when one exists. Even so, our experiences with both synthetic data and the application presented in the next section suggest that the procedure typically finds such a solution in less than 5 rounds in most cases.

\section{An illustration}

This section illustrates the computational procedure described in the previous sections using one of the few published conjoint data sets available from an actual consulting project. Although only one of the four attributes in this example is really discrete, we treat all four discrete since the original data was collected as such. The problem concerns market competition among several automobile tire manufacturers. The conjoint study, design, and actual data were published in Green (1978). The study was conducted for a nationally known marketer of automobile tires called the Alpha company. The product class of interest was steelbelted radial tires, a relatively new product at the time the study was undertaken. There were four major competitors to the Alpha Co. in the market, and are labeled as the Beta Co., the Gamma Co., the Delta Co., and the Epsilon Co.

Data for 252 male adults, between the ages of 18 and 64 , were collected from five different cities throughout the United States. As noted in Green (1978), no 
claim was made in terms of a representative sample of the entire replacement tire buying market given the sampling procedure utilized in central shopping areas with paid volunteers. Part B of the questionnaire (the entire study contained five parts or sections) was designed to measure respondents' subjective trade-offs among various attributes of a tire purchase situation - tread mileage, brand name, tire price, and convenience of the sales outlet. Table 1 shows features and levels for the full profile conjoint analysis conducted for this steel-belted radial tire market. In the table, we see that there are five competing companies and that each has three attributes with five levels to manipulate.

The study used a Graeco-Latin Square design to produce 25 full profile cards, and 252 respondents were asked to sort the cards into 5 piles designated from "Highly likely to buy" ( (core $=5$ ) to "Highly unlikely to buy" ( order to estimate part-worths of the utility function as in equation (4), a dummy variable regression was performed for each respondent. The resulting average $R^{2}$ over respondents was 0.8623 , with a range of $0.65-1.00$. Table 1 also shows a "pooled" regression analysis using the mean preference of all 252 respondents. As the table aptly denotes, tread mileage and price appear to be the most significant factors in this aggregate analysis. As expected, utility is monotone increasing with tread mileage, and monotone decreasing with price. The driving time to a store and the brand names are not statistically significant at the aggregate level.

Table 1. Tire features and conjoint estimation from the pooled sample

\begin{tabular}{|c|c|c|c|c|c|}
\hline Attributes & Code & Levels & $\operatorname{Cost}(\$)^{\prime \prime}$ & Part-worth & Sig \\
\hline \multirow[t]{5}{*}{ Advertised Tread Mileage } & 1 & 30,000 miles & 10 & 0.000 & \\
\hline & 2 & 40,000 miles & 20 & 0.212 & 0.0722 \\
\hline & 3 & 50,000 miles & 26 & 0.343 & 0.0028 \\
\hline & 4 & 60,000 miles & 30 & 0.562 & 0.0006 \\
\hline & 5 & 70,000 miles & 32 & 0.732 & 0.0001 \\
\hline \multirow[t]{5}{*}{ Price per Tire } & 1 & $\$ 40$ & $N / A$ & -0.000 & \\
\hline & 2 & $\$ 55$ & $\mathrm{~N} / \mathrm{A}$ & -0.450 & 0.0023 \\
\hline & 3 & $\$ 70$ & $\mathrm{~N} / \mathrm{A}$ & -1.228 & 0.0001 \\
\hline & 4 & $\$ 85$ & $\mathrm{~N} / \mathrm{A}$ & -1.466 & 0.0001 \\
\hline & 5 & $\$ 100$ & $N / A$ & -1.836 & 0.0001 \\
\hline \multirow{5}{*}{$\begin{array}{l}\text { Average Driving Time } \\
\text { from Home to Store }\end{array}$} & 1 & $10 \mathrm{~min}$. & 10 & 0.000 & \\
\hline & 2 & $20 \mathrm{~min}$. & 8 & -0.469 & 0.6518 \\
\hline & 3 & $30 \mathrm{~min}$. & 6 & -0.176 & 0.8646 \\
\hline & 4 & $40 \mathrm{~min}$. & 4 & -1.250 & 0.2467 \\
\hline & 5 & $50 \mathrm{~min}$. & 2 & -1.523 & 0.1662 \\
\hline \multirow[t]{5}{*}{ Company Name } & 1 & Alpha & 25 & 0.000 & \\
\hline & 2 & Beta & 22 & 0.182 & 0.1134 \\
\hline & 3 & Gamma & 19 & 0.024 & 0.8206 \\
\hline & 4 & Delta & 16 & 0.180 & 0.1169 \\
\hline & 5 & Epsilon & 13 & 0.094 & 0.3855 \\
\hline Intercept & & & & 3.146 & 0.0001 \\
\hline
\end{tabular}

"Hypothetical Cost 
At the individual level, however, a number of respondents have significant coefficients for these variables with heterogeneous utility functions.

As for the cost function, we used a set of hypothetical costs as shown in table 1 since they were not specified in Green (1978). The hypothetical scenario used in this equilibrium analysis for now is that each firm produces only one brand name tire, and determines the quality of tires (in mileage), price, and tire outlet location. To compute a Nash equilibrium in pure strategy, the sequential tatonnement process was used. The order of play was arbitrarily given as presented in table 1 (i.e., in alphabetical order). In an actual application, however, managerial judgment would be necessary to determine the order of brand repositioning. Table 2 shows the (arbitrary) initial brand positions we used.

First, we report the basic equilibrium solution under the above scenario. Table 3 shows the results of the iterative procedure that leads to the equilibrium solution. This equilibrium solution was found with less than two rounds of sequential tatonnement process. The resulting equilibrium shares and profits from the 252 respondents are also shown in table 3 . Here, Alpha maintains its high quality, price, and convenience. Beta and Delta turn to high quality and high price, but moderate convenience, while both Gamma and Epsilon produce low quality, low price tires capturing larger shares than others. As expected, a high quality brand commands a higher price, and vice versa. The differences in profits reflect the brand-specific hypothetical cost, which is the highest for Alpha and the lowest for Epsilon (see table 1).

In a sequential iteration, the order of play should make a difference. An intuitive presumption would be that the first player always has the upper hand. Yet, reversing the order of play (i.e., from Epsilon to Alpha), table 4 shows that it is not

Table 2. Initial brand positions

\begin{tabular}{llcc}
\hline Brand & Mileage(miles) & Price(\$) & Distance(min.) \\
\hline Alpha & 70,000 & 100 & 10 \\
Beta & 60,000 & 85 & 20 \\
Gamma & 50,000 & 70 & 30 \\
Delta & 40,000 & 55 & 40 \\
Epsilon & 30,000 & 40 & 50 \\
\hline
\end{tabular}

Table 3. Sequential equilibrium: Base case (for codes, see Table 1)

\begin{tabular}{lrrrrr}
\hline Iter & Alpha & Beta & Gamma & Delta & Epsilon \\
\hline 1 & 5511 & 1512 & 5553 & 5534 & 1255 \\
2 & 5511 & 5532 & 1253 & 5534 & 1255 \\
Share & 0.16 & 0.17 & 0.24 & 0.17 & 0.26 \\
Profits & 1730.78 & 1990.60 & 1997.22 & 2197.08 & 2261.80 \\
\hline
\end{tabular}


Table 4. Sequential equilibrium: Reverse order of play

\begin{tabular}{lccccc}
\hline Iter & Epsilon & Delta & Gamma & Beta & Alpha \\
\hline 1 & 5535 & 1254 & 1253 & 5532 & 5511 \\
2 & 5535 & 1254 & 1253 & 5532 & 5511 \\
Share & 0.16 & 0.27 & 0.24 & 0.17 & 0.16 \\
Profits & 2177.55 & 2267.09 & 1943.37 & 1971.25 & 1726.18 \\
\hline
\end{tabular}

necessarily true. In table 4, three brands (Alpha, Beta and Gamma) maintain the same position as in table 3: the positions are optimal regardless of the order of play. However, the roles of Delta and Epsilon are reversed: Delta is the low-end brand this time. Comparing the two tables, we find that the profit levels are not much different. In fact, the profit of Epsilon has slightly decreased by being the first player. Nor does Alpha lose much by being the last one to move. Perhaps, this is due to the lack of foresight in our model. Since firms have no information about others' next strategies, the first player loses its advantage unless consumer preferences are highly concentrated, in which case the first to position captures the largest share. Otherwise, the subsequent players have relative advantages since the strategies of the preceding players have been revealed. In the absence of foresight, therefore, the advantage of being the first player depends on the balance between competitive information and the degree of concentration of consumer preferences. Modeling foresight in our context would be a challenging task for future research.

Finally, changes in the cost structure may play a role in shaping an equilibrium. Intuitively, we expect higher profits as cost decreases. Also, Choi, DeSarbo, and Harker (1990) have shown that equilibrium prices move in the same direction as cost levels in a continuous space. However, our simulation results show that these are not always the case in a discrete strategy space. We solved the model by changing costs within \pm 2 from the base case. All equilibria were found in less than three rounds.

Although the equilibrium solutions are not directly comparable because of the potential local optimality, the profit levels were remarkably consistent across the cost range for most brands. It appears that equilibrium profits in a discrete game is not very sensitive to small cost changes. Yet, low-priced brands continue to have larger shares than high-priced brands with generally better features. It is also interesting to note that, in most cases, there are two low quality and three high quality brands. Perhaps it indicates that this is the optimal structure of industry differentiation under the current assumptions.

\section{Conclusion}

Conjoint analysis has seen countless successful applications in product and service design problems. While many researchers have recognized the need for the 
methodologies to model competitive reactions in this context, little work has been done due to the discrete nature of variables in most conjoint measurement procedures. In this paper, we employed an optimization approach to numerically compute Nash equilibria under either profit or market share objectives. While our proposed methodology relies heavily on numerical computation in finding competitive strategies, compared to the choice simulator-based methods, our procedure needs to evaluate only a small subset of potential product designs to compute an equilibrium solution.

We found that the initial positions are important in shaping the equilibrium since all strategies are conditioned on the others' previous positions. The first player in the sequential tatonnement does not always have an advantage over the subsequent players, particularly when there is no foresight in the competitors' future strategies. Minor changes in costs play a relatively small role in equilibrium prices and profits. Also, lower costs do not necessarily result in higher profits in a discrete game.

Unlike most marketing studies of competition that deal with continuous variables, there are no analytical closed-form solutions for our problem. The existence of an equilibrium has to be established by actually computing one. The uniqueness of an equilibrium is even more difficult to prove. Even so, our procedure attempts to provide valuable insights into the nature of product competition when the variables are categorical. Since our paper seeks to find Nash solutions, these methodologies may not directly provide actual "optimal" solutions. Rather, these approaches suggest the results of a competitive process assuming all other players are equally rational. Still, the insights gained from these models can be used in managerial decision making of product strategy.

There are other related questions yet to be answered in future research: Which equilibrium is most likely to occur when there are multiple equilibria? Can they jump from one equilibrium to another easily? How does the analyst deal with the positioning of multiple brands from multiple manufacturers that compete in different market segments (see Kohli and Sukumar 1990)? How does one model foresight and dynamic (changing) consumer preferences that change over time/offerings? How do solutions obtained with assumptions of sequential moves differ, in general, from those obtained assuming simultaneous moves? What type of probabilistic statements can be made to summarize the various solutions obtained after a particular attribute/cost change? We believe that the methodology will become a cornerstone for the development of more comprehensive models that may address these issues in the future.

\section{References}

Carpenter, Gregory S. (1989). "Perceptual Position and Competitive Brand Strategy in a TwoDimensional, Two-Brand Market," Management Science 35, 1029-1044.

Choi, S. Chan. Wayne S. DeSarbo, and Patrick T. Harker. (1990). "Product Positioning under Price Competition," Management Science 36, 175-199. 
Choi, S. Chan, Wayne S. DeSarbo, and Patrick T. Harker. (1992). "A Numerical Approach to Deriving Long-Run Equilibrium Solutions: A Research Note," Forthcoming, Management Science $38,75-86$.

DeSarbo, Wayne S., and Vithala Rao. (1986). "A Constrained Unfolding Model for Product Positioning," Marketing Science 5, 1-19.

DeSarbo, Wayne S., M. Wedel, M. Vrien, and V. Ramaswamy. (1992). "Latent Class Metric Conjoint Analysis," Marketing Letters 3, 273-288.

Dobson, Gregory, and Shlomo Kalish. (1993). "Heuristics for Pricing and Positioning a ProductLine Using Conjoint and Cost Data," Management Science 39, 160-175.

Friedman, James. (1990). Game Theory with Applications to Economics (2nd Ed.). New York: Oxford University Press.

Green, Paul E. (1978). Analyzing Multivariate Data. Hinsdale, IL: Dryden Press.

Green, Paul E., J. Douglas Carroll, and Stephen M. Goldberg. (1981). "A General Approach to Product Design Optimization via Conjoint Analysis," Journal of Marketing 45, 17-31.

Green, Paul E., and Wayne S. DeSarbo. (1979). "Componential Segmentation in the Analysis of Consumer Tradeoffs," Journal of Marketing Research 43, 83-91.

Green, Paul E., and Abba M. Krieger. (1985). "Models and Heuristics for Product Line Selection," Marketing Science 4, 1-19.

Green, Paul E., and Abba M. Krieger. (1992). "An Application of a Product Positioning Model to Pharmaceutical Products," Marketing Science 11, 117-132.

Green, Paul E., and Vithala R. Rao. (1971). "Conjoint Measurement for Quantifying Judgmental Data," Journal of Marketing Research 8, 355-363.

Green, Paul E., and V. Srinivasan. (1978). "Conjoint Analysis in Consumer Research: Issues and Outlook," Journal of Consumer Research 5, 103-123.

Green, Paul E., and V. Srinivasan. (1990). "Conjoint Analysis in Marketing Research: A Review of New Developments," Journal of Marketing 54, 3-19.

Kohli, Rajeev, and R. Sukumar. (1990). "Heuristics for Product-Line Design Using Conjoint Analysis," Management Science 36, 1464-1478.

Rubinstein, Ariel. (1991). "Comments on the Interpretation of Game Theory," Econometrica 59. 909-924.

Shocker, Alan D., and V. Srinivasan. (1979). "Multiattribute Approaches for Product Concept Evaluation and Generation: A Critical Review," Journal of Marketing Research 16, 159-180.

Wittink, Dick R., and Philippe Cattin. (1989). "Commercial Use of Conjoint Analysis: An Update," Journal of Marketing 53, 91-96.

Zufryden, Fred S. (1982). "Product Line Optimization by Integer Programming," Proceedings of the Annual Meeting of ORSA/TIMS, San Diego. 